Mavi Atlas, 6(1)/2018: 308-325. Araştırma Makalesi | Research Article

Makale Geliș | Received: 13.02.2018

Makale Kabul | Accepted: 25.03.2018

DOI: 10.18795/gumusmaviatlas.418999

\title{
Ŏguzhan EKİNCí
}

\author{
Dr. Öğr. Üyesi \\ Erzurum Teknik Üniversitesi, Edebiyat Fakültesi, Sosyoloji Bölümü, Erzurum-Türkiye \\ Technische Universität Erzurum, Philosophische Fakultät, Fachbereich Soziologie, Erzurum-Türkei \\ oguzhan.ekinci@erzurum.edu.tr
}

\section{Staat und Souveränität: Von der Antike bis zur Neuzeit - Eine Skizze}

\section{Zusammenfassung}

Thema des vorliegenden Aufsatzes ist die Konzeption des Souveränitätsbegriffs aus diachronischer Sicht beginnend mit der Antike anhand der platonischen und aristotelischen Staatsauffassung über die neuzeitliche Auslegung von Hobbes und Bodin bis hin zu dem modernen Staatsrechtler der Weimarer Republik Carl Schmitt. In der Auseinandersetzung mit der Schaffung einer politisch staatlichen Ordnung und einer Abgrenzung nach Außen, zeigt sich, dass auch in der heutigen politischen Situation, das Freund-Feind-Unterscheidung und die Erhaltung einer staatlichen Souveränität als Schlüsselkonzept zum Verständnis des staatlichen Handelns fungiert.

Schlüsselwörter: Staat, Souveränität, Politische Ideengeschichte, Carl Schmitt.

\section{Devlet ve Egemenlik: Antik Çağdan Modern Zamanlara - Bir Eskiz}

\section{$\ddot{O} z$}

$\mathrm{Bu}$ makale, devlet ve egemenlik kavramlarını merkeze alarak Antik Çağ’1 temsilen Platon ve Aristoteles'in, Yeniçağ'1 temsilen Hobbes ve Bodin'in ve moderniteyi temsilen de Carl Schmitt'in siyasal düşüncelerini diakronik olarak tartışmaktadır. Çünkü bu kavramlar, bir devletin siyasal düzenini kurmasında, dışa dönük olarak egemenliğini iddia etmesinde ve bunları korumak için dost-düşman ayrımı yapmasında eskiden olduğu gibi günümüzde de son derece önemli bir yere sahiptir. Bu bakımdan devletlerin bazı politikalarını, anlamak, yorumlamak ve kavramsallaştırmak için bu kavramların yakından irdenlenmesi faydalı olacaktır.

Anahtar Kelimeler: Devlet, Egemenlik, Siyasal Düşünceler Tarihi, Carl Schmitt. 


\title{
I. Einleitung
}

\begin{abstract}
"Worüber müßten wir uns wohl streiten und $\mathrm{zu}$ was für einer Entscheidung nicht kommen können, um uns $\mathrm{zu}$ erzürnen und einander feind zu werden? Laß mich es aussprechen, und überlege, ob es wohl dieses ist: das Gerechte und Ungerechte, das Edle und Schlechte, das Gute und Böse. Sind nicht dies etwa die Gegendstände, worüber streitend und nicht zur völligen Entscheidung gelangend einander feind werden, sooft wir es werden, du und ich sowohl als auch alle übrigen Menschen?" (Platon, Euthyphron 7 c-d).
\end{abstract}

Wenn es darum geht, das Wesen und den Aufgabenbereich des Staates aus dem Blickwinkel der politischen Theorie zu erhellen, wird seit Bodin der Staat als jene innehabende Instanz charakterisiert, den der Begriff der 'Souveränität' zugesprochen werden kann und muss.

Souveränität verstanden als auctoritas - potestas im Sinne einer obersten Befehlsgewalt über die feste Bezugsgröße eines gesicherten territorialen Raumes (Staatsgebiet) mit einer expliziten Benennung (Name) und einer spezifischen Bevölkerung (Staatsvolk) (Jellinek 1959: 394), muss als solche um mit Luhmann zu sprechen auf jene politische Ordnung appliziert werden, die sich als Letztinstanz politischen (als kollektiv bindenden) Entscheidens versteht, i. e. dem Staat (Luhmann 2000: 197-198).

Begreift man diesen zentralen Terminus der Souveränität als juristische Determinante (frz. Souverainetè, aus lat. superanus = darüber befindlich, überlegen) wird hier das Bild einer politischen Ordnung skizziert, die sich in ihrem Recht auf Selbstbestimmung als personalisierte Handlungsinstanz versteht, die einer Fremdbestimmung entgegengestellt ist. Während Bodin diese Handlungsmacht auf den Souverän (Monarch) einer durch Glaubenskriege gequälten französischen Nation applizierte (monarchische Souveränität), charakterisieren sich die modernen Staaten als politische Ordnungssysteme, die die Souveränität ihrer jeweiligen Staatsvölker repräsentieren (Volkssouveränität).

Zwischen diesen beiden äußersten Formen der Auslegung, wie sich Souveränität als Handlungsmacht formiert, liegen knapp 500 Jahre europäischer politischer 
Tradition, und es scheint auf der Hand, daß ein feudales- durch das monarchische Prinzip -gekennzeichnete Gesellschaftssystem einer anderen Staats- und Souveränitätsdefinition zugrunde liegt, als der moderne -durch Parteien und Interessengemeinschaften vertretene föderale- Staat des 20. und des metamodernen Staates des 21. Jahrhundert, der im Zeitalter der fortschreitenden Globalisierung und Technisierung auch innerhalb der europäischen Tradition weitestgehend nicht mehr nur dem Prinzip des souveränen Staates sondern auch dem der Suzerität folgt, in dem er einen Teil des autonomen nationalstaatlichen Handlungsvollzugs z.B. an die europäische Gemeinschaft überantwortet (Währungs-, Grenz-Zollsystem/ militärisches Oberkommando/ 'ius belli' sowie Kultur- und Sprachpolitik). ${ }^{1}$

Ausgehend von dem durch Bodin geprägten klassischen Souveränitätsbegriff, der diese dem Monarchen in einer von Krisen gekennzeichneten historischen Epoche überantwortete, soll dieser quasi als Hintergrundfolie auf die Situation der gegenwärtigen 'modernen' Staaten appliziert werden in einer Periode staatswissenschaftlichen Denkens, in der nicht mehr von den Bedingungen der Möglichkeit zu staatlicher (i.e. monarchischer) Souveränität gesprochen wird wie bei Bodin, sondern vielmehr von jener im Zeitalter der Politik der Parteiendemokratie und des Liberalismus als fortschreitender Individualisierung auf der gesellschaftlichen und politischen Ebene im Innern und fortschreitender Zentralisierung der europäischen Staatsgemeinschaften auf außenpolitischer Ebene des pessimistischen Diktums der „prekären Staatlichkeit” sowie des Zweifels an der Möglichkeit zur Wahrung staatlicher Souveränität überhaupt geführt haben. Kennzeichnend und charakteristisch für den Kulturpessimismus ob des fortschreitendes Verlust staatlicher Souveränität in der Moderne ist bekanntermaßen das Oeuvre des Juristen und Staatrechtlers Carl Schmitt. ${ }^{2}$ Der vorliegende Aufsatz beschäftigt sich mit dieser zentralen -material- Staaten

\footnotetext{
1 So konstatiert Mäder: "Staat und Souveränität sind zwei Begriffe, die in ihrer geschichtlichen Entwicklung und rechtlichen Zuordnung aufeinander verweisen und [...] sich gegenseitig bedingen" (Mäder 2007: 35).

2 “[...] der faszinierendste, politisch umstrittene aus heutiger Sicht bedeutendste deutscher Staatsrechtler des Zwanzigsten Jahrhunderts” (Mäder 2007: 47).
} 
konstituierenden Begriff der Souveränität und der vielbeschworenen Krise des modernen Staates aus der Perspektive der Staatstheorie Carl Schmitts.

\title{
II. Souveränität als Begriff der politischen Ideengeschichte von der Antike
}

\section{bis zur Neuzeit}

\begin{abstract}
"Der Gegenwart entfremdet. -Es hat große Vorteile, seiner Zeit sich einmal in stärkerem Maße zu entfremden und gleichsam von ihrem Ufer zurück in den Ozean der vergangenen Weltbetrachtungen getrieben $\mathrm{zu}$ werden. Von dort aus nach der Küste blickend, überschaut man wohl zum ersten Male ihre gesamte Gestaltung und hat, wenn man sich ihr nährt, den Vorteil, sie besser zu verstehen als Die, welche sie nicht verlassen haben." (Friedrich Nietzsche, Menschliches, Allzumenschliches).
\end{abstract}

Im folgenden sei, bevor zum eigentlichen Kernthema des Aufsatzs vorgedrungen wird, der Begriff der Souveränität aus historischer Perspektive umrissen, schließlich hat das politische Denken und die damit zusammenhängende Reflexion über das Wesen des Staates und über das, was als Kennzeichnung von Souveränität definiert wurde nicht erst im letzten Jahrhundert begonnen, sondern reicht knapp zwei tausend Jahre zurück. ${ }^{3}$

Der in der Einleitung konstatierte Kulturpessimismus Carl Schmitts über den wachsenden Verlust staatlicher Souveränität im Zeitalter des entpolitisierenden Liberalismus beginnt so zu sagen schon bei Platon, der das kennzeichnende Moment einer maßlosen Freiheit die Demokratie zu Grunde richten sieht (Platon 1998: 564). So fürchtet Platon in der "Unersättlichkeit des Dranges nach Freiheit" das Umschlagen der Demokratie in Tyrannei (Zippelius 2003: 19). Platons Auffassung über die Aufgaben des Staates kulminiert in dem Prinzip, dass “der Staat das Zusammenleben der Menschen in der bestmöglichsten Weise zu ordnen habe“ (Zippelius 2003: 20). In einer Art antiker arbeitsteiligen Gesellschaft sollte jedem die Möglichkeit gegeben sein, gemäß seiner natürlichen Anlagen am Leben der Gemeinschaft mitzuwirken (Platon 1998: 617d- 618e). Staatsideal für Platon war der aristokratische Dreiständestaat. "Der

\footnotetext{
${ }^{3}$ Der erste Beleg zu “Souveränität” stammt aus dem Jahre 1120 (Quaritsch 1986: 13).
} 
Souverän“" als Machthaber im Staat kann nur jener sein, der von der Erkenntnis und der Liebe zur Wahrheit und zu dem was gut ist, getrieben wird. Während also die Forderung dass, "entweder die Philosophen Könige in den Staaten werden oder die, welche jetzt Könige und Herrscher heißen, echte und gründliche Philosophen werden" die Bedingungen zum Staatsmännischen Tun umreißt, formuliert Platon die Auffassung, daß staatliche Souveränität, elitärer Stand und philosophisches Denken zum Nutzen des Gemeinwohls notwendig zusammenfallen müssen (Platon 1998: 473 c-d). Souveränität in diesem Sinne bedeutet das Innehaben einer Kontrollinstanz, die sich auf sämtliche Bereiche des gemeinschaftlichen Lebens auszudehnen hat, bis hin zur damit verbundenen Ausbildung einer Bürgertugend und der asketischen Selbstzucht des Polisbürgers.

Ähnlich wie sein Lehrer Platon begreift auch Aristoteles, dass Vernunft und Gerechtigkeit bei nur wenigen zu finden sei und formuliert daher, dass "wenn einer an Tugend die anderen weit überragte, möchte es richtig sein, dass er herrscht" (Aristoteles 1995: 1284b). Dabei jedoch muß die Gemeinschaft derjenigen, die die Gemeinschaft bilden nicht von überdurchschnittlicher Bildung der Menschen oder einem Wunschbild der Verfassung ausgehen. Grundlage eines stabilen Verfassungsstaates sei vielmehr, daß der Teil der politischen Gemeinschaft, der die Erhaltung des Staates will, immer stärker sein muß als der, der sie nicht will. Notwendig dabei ist, dass es für die Regierenden wichtig ist, daß alle in gleicher Weise abwechselnd regieren und regiert werden (Zippelius 2003: 34). Souveränität wird hier aufgelöst in eine Staatsmacht, die sich im Fluß und ständigen Werden einer auf das Gemeinwohl und die Gerechtigkeit ausgerichteten Gemeinschaft konzentriert. ${ }^{4}$

Mit der Entstehung des Christentums tritt der Gottesgedanke explizit in die Theorieentwürfe politischen Denkens ein Augustinus entwickelt die Zwei- StaatenLehre (Civitas dei) und die Zwei- Reiche- Lehre bringt zum Ausdruck, das die staatliche

\footnotetext{
${ }^{4}$ Natürlich kann nicht vom Begriff als solchem ausgegangen werden, da dieser ja erst bei Bodin auftaucht, nichtsdestotrotz kommt in den Werken der Antike eine Auffassung über der Sinn und das Wesen der staatskonstituierenden Macht zum Tragen, die hier exemplarisch skizziert wird um zum Souveränitätsbegriff Carl Schmitts überleiten zu können.
} 
Ordnung, wie tyrannisch oder verwerflich sie auch sein mag als Ausdruck eines gottgewollten Schöpfungsplans gedacht werden muß (Geerlings 2002: 72).

Wegbreiter jedoch des Souveränitätsbegriffs als Machtkompetenz zur Sicherung einer gesellschaftlichen Lebensordnung per Staatsmacht war der französische Jurist Jean Bodin (1530- 1596), der explizit in seinem Werk (Six livres de la république, 1576 I, Kap. I), die Souveränität als Ausdruck des Wesens des Staates begreift.

In einer vom Kampf um Hoheitsrechten geprägte historische Epoche, in der es darum ging Frankreich entgegen den Ansprüchen durch dem Papst und Kaiser die Oberherrschaftsansprüche dem König zuzuerkennen, definiert Bodin Souveränität als das Vermögen “alle Untertanen ohne deren Zustimmung Gesetze aufzuerlegen” (Bodin 1981: 222). So formuliert er als das Kennzeichen der Souveränität die absolute Kontrollgewalt als potestas absolutas und als solche frei von hemmenden anderen Instanzen sowohl im Innern als auch im Außen, als auch die Unteilbarkeit der Souveränität als solchen.

"Diese Gewalt Gesetze zu erlassen oder aufzuheben, umfaßt zugleich alle anderen Rechte und Kennzeichen der Souveränität, so daß es streng genommen nur dieses eine Merkmal der Souveränität gibt. Alle anderen Souveränitätsrechte sind darunter subsumierbar: über Krieg und Frieden, das Recht der letzten Instanz, das Ernennungsund Absetzungsrecht für die obersten Beamten, das Besteuerungsrecht, das Begnadigungsrecht, das Münzrecht und die Festsetzung des Geldwerts, das Recht auf Treueide der Untertanen und Vasallen. Dies sind die wahren Kennzeichen der Souveränität, die zusammengefaßt sind in der Gesetzgebungsgewalt gegenüber allen Untertanen insgesamt und einzelnen von ihnen" (Bodin 1981: 241).

Souveränität im Sinne Bodins wird zur zentralen Machtinstanz, ${ }^{5}$ die nur durch die Gebote Gottes, natürliche Gesetze sowie allgemeine Rechtsgrundsätzen (plusivies loix

\footnotetext{
5 "Unter Souveränität ist die dem Staat eignende absolute und zeitlich unbegrenzte Macht zu verstehen." (Bodin 1981: 205).
} 
humaines, communes a tous peuples) Schranken gesetzt sind. ${ }^{6}$ Aktives Widerstandsrecht oder Einwirkung auf den Willen des Souveräns bleibt untersagt: “Alle Stände sind dem König Untertan, der weder ihren Rat sucht, noch sich nach ihrem Willen richtet“". Ebenso umstritten bleibt die Unteilbarkeit der Souveränität sowie die Möglichkeit gemischter Verfassungen- der einzige denkbare politische Modus ist und bleibt für Bodin die Monarchie: "Das hervorstehende Merkmal des Staates, das Souveränitätsrecht, kann es im strengen Sinne nur in einer Monarchie geben, denn niemand als nur ein einziger kann im Staat souverän sein (Bodin 1981: 111).”

Diametral entgegengesetzt $\mathrm{zu}$ platonischer und der aristokratischer Staatskonzeptionen bleibt Bodin einem absolutistischen Prinzip verhaftet. Doch schon hier zeigt sich ein besonderes Motiv, das später auch im Schmittianschen Denken auftauchen wird, der davon ausging, daß alle modernen Staatsbegriffe immanentisierte theologische Begriffe seien: Die Unteilbarkeit der Souveränität, die Fügung vor dem Willen des Alleinherrschenden, der keine imperium soci: neben sich haben kann, all diese Motive scheinen dem christlichen Monotheismus auf staatliche politische Ebene transportiert, entnommen.

Ein weiterer politischer Theoretiker, der den Souveränitätsgedanken bei Carl Schmitt maßgeblich geprägt hat und die Möglichkeit politischer Ordnung in den Zeiten von Bürger und Konfessionskriegen formulierte, ist Thomas Hobbes.

Doch während das antike Menschenbild durchweg positiv gezeichnet ist (im aristotelischen Sinne entfaltet sich die soziale Gemeinschaft zum Gemeinwohl aller auf der Basis der Verwirklichung positiver Fähigkeiten und Charaktereigenschaften) zeichnet Thomas Hobbes ein durchweg negatives Menschenbild, das er mit dem Diktum "homo homini lupus" beschreibt. Im Naturzustand als prä-staatliche

\footnotetext{
6 "Den Gesetzen Gottes und der Natur sind alle Fürsten der Erde unterworfen und es steht nicht in ihrer Macht sich über sie hinwegzusetzen, ohne sich eines Majestätsverbrechens an Gott schuldig zu machen und damit offen Gott den Krieg zu erklären.” (Bodin 1981: 98).

7 "Der Mensch ist des Menschen Wolf."
} 
Lebensform ohne die Kontrollinstanz eines Ordnung gewährenden Souveräns ist der Einzelne seinem Nebenmenschen schutzlos ausgeliefert. ${ }^{8}$

Wie kann also eine staatliche Ordnung hergestellt werden vor dem Hintergrund der von menschlichen Trieben und Leidenschaften besessenen Einzelwillen? Der Leviathan, das alttestamentarische Seeungeheuer und als solches Symbol für übermenschliche Stärke aber auch der politischen Ratio/Vernunft ist es, der die Einzelwillen unter dem Willen des Souveräns eint. Im Sinne einer Konstruktion, von den Menschen selbst geschaffen, als Maschine, Konstrukt zu ihrem eigenen Nutzen, überantworten die einzelnen per Vertrag ihren Eigenwillen demjenigen des Souveräns (Mäder 2007: 60). Wie auch schon bei Bodin, so konzipiert Hobbes die Souveränität des Leviathan als unteilbar (Hobbes 2005: 153). Doch während bei Bodin die Souveränität dem Monarch überantwortet wird, so ist der Leviathan der Ausdruck eines per Vertragschluß konstituierten Gemeinschaftswesen, das eine Verkörperung mittels Repräsentation erfährt (Hobbes 2005: 145). Durch die Personalisierung eines stellvertretenden Souveräns, wobei Repräsentation hier nicht die bloße Stellvertretung meint, sondern vielmehr zur Sichtbarmachung eines ideell Gedachten, die Subsumtion der Einzelwillen unter eine übergeordnete Instanz, führt. Souveränität meint hier also die Übermacht und das Vermögen den Herrschaftsvertrag mittels Einsicht herstellen zu können. Jedoch auch Hobbes betont hierbei, daß im Notfall der Souverän, schon wie bei Bodin, auch gegen den Willen der Bürger Entscheidungen treffen muß als Handlung im Sinne des Gemeinwohls unter dem Prinzip der Aufrechterhaltung der souveränen Handlungsmächtigkeit des Souveräns (Hobbes 2005: 174, 187, 205).

Somit untersteht der Souverän nicht dem Gesetz selbst, da durch diesen die politische Ordnung erst fundiert wird, was hier paradox und aus modernen Sicht "antidemokratisch" anmutet, muß jedoch im historischen Kontext gelesen werden: die

\footnotetext{
${ }^{8}$ Als Naturzustand soll im folgenden eine menschliche Seinsweise verstanden werden, die sich durch Vor-Staatlichkeit, d.h. durch das Fehlen einer übergeordnete Instanz auszeichnet. Es ist fraglich, ob sich ein derartiger Zustand faktisch historisch nachweisen kann. In diesem Sinne ist dieser Zustand idealiter gedacht. MacPherson betrachtet das Hobbessche Menschenbild aus Ausdruck der Strukturen der englischen, bürgerlichen Gesellschaft des 17. Jahrhunderts (MacPherson 1962).
} 
hobbessche Staatstheorie entstand vor dem Hintergrund einer im Bürger- und Religionskriegen zerrissenen englischen Nation.

Festzuhalten gilt, daß Hobbes wie auch schon Bodin die Bedingungen der Möglichkeit politischer Ordnung vor dem Angesicht eines als den Menschen, sein Eigentum und Leben im concreto bedrohenden Naturzustand zu umreisen sucht. Das negative Menschenbild, das hier skizziert wird, führt $\mathrm{zu}$ der Einsicht, daß die Herrschaftsbedürftigkeit des Menschen anthropologische Grundkonstante $\mathrm{zu}$ sein scheint: Souveränität als Handlungs- und Entscheidungskompetenz ist das Vermögen als Herrschaftsinstanz die divergenten Einzelwillen zu subsumieren um eine politische Lebensordnung herbeizuführen.

\title{
III. Carl Schmitt: Staat und Souveränität
}

\begin{abstract}
"Praktisch hat eine Jurisprudenz, die sich an den Fragen des täglichen Lebens, und der laufenden Geschäfte orientiert, kein Interesse an dem Begriff des Souveränität. Auch für sie ist nur das Normale das Erkennbare und alles andere eine 'Störung'." (Carl Schmitt, Politische Theologie).
\end{abstract}

Als Verfassungsrechtler und Jurist, in den Zeiten der Weimarer Republik, formulierte Carl Schmitt dem Souveränitätsbegriff in einer historischen Situation, in der er die politischen Ordnung gefährdet sah. ${ }^{9}$ Carl Schmitts offensichtliche Aversionen gegen einen politischen Pluralismus, der die Souveräntität des Staates unterbindet, stellt eines der Grundcharakteristika seiner politischen Philosophie dar. Verträge politischer Natur sind aus seiner Perspektive dazu da, die Souveränität des Staates zu unterbinden und in seiner Handlungsfähigkeit stark einzuschränken. In diesem Sinne begreift Carl Schmitt politische Verträge als fundamentale Gefahr für die politische Souveränität des Staatets (Schmitt 1996: 13). Anders als bei Hobbes und Bodin entscheidet nicht mehr die Möglichkeit der Gestaltung einer politischen Lebensordnung über die Zurechnung von Souveränität, also inwieweit diese Ordnung hergestellt zu werden vermag, sondern

\footnotetext{
${ }^{9}$ Zur historischen Situationen vgl. Rosenberg, Arthur (1983). Entstehung und Geschichte der Weimarer Republik, Frankfurt am Main: Europäische Verlagsanstalt.
} 
gerade der Ausnahmezustand und die Entscheidung ob die jeweilige konkrete politische Situation als solche definiert werden muß und die Entscheidung wie gehandelt werden soll, ist das maßgebliche Charakteristikum der souveränen Handlungskompetenz (Vad 1996: 20).

Wer jedoch trifft laut Schmitt diese Entscheidung? Wer ist das Subjekt der Souveränität? Der Verfassungsjurist und Rechtsstaatler, quasi das Recht per se kann nur Entscheidungen aus den Prämissen des gesetzten Rechts selbst heraus treffen. Die Jurisprudenz, so Schmitt hat keine Interesse an den Fragen der Souveränität, Entscheidung werden aus der Norm heraus getroffen. Der Souveränitätsbegriff generiert somit zum Grenzfall, also zu einem Grenzbegriff der äußersten Sphäre. Die Entscheidung über die Ausnahme wird zur "Entscheidung im eminenten Sinne". Während die Entscheidung im juristischen Sinne restlos aus dem Inhalt einer Norm abgeleitet werden muß, so übersteigt die Entscheidung über die -rechtliche nicht gesetzte Ausnahme- die Immanenz des positiven Rechts.

Der Streit um die Anwendung des Rechts wird zum Konfliktfall und so ergibt sich für Schmitt das abstrakte Schema: daß Souveränität die höchste, nicht abgeleitete Herrschaftsmacht beinhaltet, denn die Norm ist schlichtweg nicht fähig die Ausnahme zu begründen. Souveränität ist somit der legitime (jedoch nicht durch positives Recht legitimierte) Anspruch im Ausnahmefall das Recht zu suspendieren. Der Staat schafft die Rechtsordnung, kann diese jedoch im äußersten Grenzfall zur Verteidigung derselbigen außer Kraft setzen.

So ist, laut Schmitt, jegliches Recht Situationsrecht und der Souverän als solchem kann durch die systemische Punkte definiert werden als jener der die „Garantie des Grenzen" durchsetzt, die "Schaffung von Totalität" zu leisten imstande ist sowie derjenige, der über das "Entscheidungsmonopol” verfügt: "Im Ausnahmefall offenbart das Wesen der staatlichen Souveränität sich am klarsten" (Schmitt 1996: 19). Die Negation des "Normalfalles", nämlich der potentielle oder tatsächliche "Ausnahmefall" steht im Zentrum seines Denkens, weil "die Regel von der Ausnahme lebt" (Schmitt 1996: 11-12). Denn "das Normale beweist nichts, die Ausnahme beweist alles; sie 
bestätigt nicht nur die Regel, die Regel lebt überhaupt nur von der Ausnahme. In der Ausnahme durchbricht die Kraft des wirklichen Lebens die Kruste einer in Wiederholung erstarrten Mechanik" (Schmitt 1996: 15). Man könnte behaupten, dass für Schmitt die Normalität das Ergebnis und die Ausnahmesituation den Weg darstellt (Lietzmann 1998: 109). So gesehen ist sein Denken polemologisch, weil auch in der Realität des Politischen, Gewalt und Krieg als Möglichkeiten vorhanden sind. In diesem Zusammenhang ist die Entscheidungsbefugnis über den Ausnahmezustand, das Hauptgebiet der politischen Souveränität (Vad 1996: 80). "Souverän ist, wer über den Ausnahmezustand entscheidet" (Schmitt 1996: 11). Insofern bestimmt die Entscheidung des Souveräns den Ausnahmezustand (Schmitt 1996: 37). Bis zur Wiederherstellung der Normalität ist der Souverän die alleinige Entscheidungsinstanz in der vorherschenden politischen, sozialen und wirtschaftlichen Ordnung (Lietzmann 1998: 110-111). So deutet Schmitt auf die reale Möglichkeit des Ausnahmefalls und meint, dass der Ausnahmefall eine "enthüllende Bedeutung” hat (Schmitt 1998: 35).

Schmitt konstatiert, daß das Entscheidende an den Ausführungen Bodins zum Souveränitätsbegriff darin liegt, daß er die Dezision in den Souveränitätsbegriff integriert hat. Doch kennzeichnend für die Situation des Souveräns in der Epoche des Liberalismus in materialiter als auch in der politischen Theorie per se läuft darauf hinaus, den Souverän als Subjekt der Souveränität zu beseitigen. Denn das Paradoxon, das diejenige Instanz, die Recht setzen kann, indem sie aber es auch gleichzeitig suspendieren kann- zeigt, daß der moderne Staat als säkularer Staat, den Kern seiner Auflösung bereits in sich trägt. Diese latent empfundene Gefahr der Auflösung der politischen Ordnung durch den Souverän ist jenes als Bedrohung empfundene Gefahrenmoment, welches die Jurisprudenz und politische Wissenschaft zu überwinden sucht, indem sie die Endscheidungsmacht als solche leugnet (Schmitt 1998: 21-22).

Schmitt schreibt: “Kelsen löst das Problem des Souveränitätsbegriffs dadurch, daß er es negiert. Der Schluß seiner Deduktion ist: "Der Souverän muß radikal verdrängt werden. In der Sache ist dies die alte liberale Negierung des Staates gegenüber dem Recht und die Ignorierung des selbständigen Problems der 
Rechtverwirklichung. Diese Auffassung hat eine bedeutende Darlegung gefunden durch H. Krabbe, dessen Lehre von der Rechtssouveränität [...] auf der These beruht, daß nicht der Staat, sondern das Recht souverän ist" (Schmitt 1996: 29-30). Und weiter: "Die moderne Staatsidee setzt nach Krabbe an die Stelle einer persönlichen Gewalt (des Königs, der Obrigkeit) eine geistige Macht. Wir leben jetzt nicht mehr unter der Herrschaft von Personen, seien es natürliche oder konstruierte (Rechts-)Personen, sondern unter der Herrschaft von Normen, geistigen Kräften. Darin offenbart sich die moderne Staatsidee" (Schmitt 1996: 29-30). "Die verschiedensten Theorien des Souveränitätsbegriffs - Krabbe, Preuß, Kelsen- [...] sind sich darüber einig, das alles persönliche aus dem Staatsbegriff verschwinden muss. Persönlichkeit und Befehl gehören für sie offenbar zusammen" (Schmitt 1996: 36-37).

Gegen Krabbe, Preuß und Kelsen jedoch beharrt Schmitt auf dem Primat der Entscheidung, daß die Dezision dem Souverän vorbehalten werden muß und er sieht in dem Versuch der Abschaffung des Souveräns nur die zur Spitze getriebene Entpolitisierung eines liberalen Verfassungsstaatesparadigmas, die das Ergebnis des neuzeitlichen Zeitalters ist, das er als Zeitalter der Entpolitisierung per se begreift: Das metaphysische Element, welches jeder politischen Ordnung vorausgeht indem sie diese erst konstituiert, indem sie diese transzendiert- wird negiert- indem die Souveränität als solche negiert und in die Positivität des gesatzten Rechts verlagert wird. Dabei jedoch ist das Politische und die damit zusammenhängende Kategorie Unterscheidung von Freund und Feind, und die damit zusammenhängende Herrschaftsbedürftigkeit des Menschen als anthropologische Grundkonstante, Voraussetzung und notwendige Vorbedingung des Staates- denn das Politische ist das Maßgebende. Da das Politische im Denken Schmitts vom Moralischen, Ökonomischen oder Religiösen geschieden ist bedeutet Feindschaft, “die Negation der eigenen Art Existenz” (Meier 1998: 27-28).

Die Schmittianische Freund-Freind Unterscheidung konstituiert eine politische Ordnung im Innern und einem Feind nach Aussen. Auf diese Weise ist der Staat dazu fähig und in der Lage, eine politische Ordnung innerhalb seines definierten Rahmens zu schaffen, in Abgrenzung von einem als feindlich empfundenen Aussen. So muss ein 
Staat in seinem Existenz ein Außen, dass es zum bekampfen und ein Inneres, dass es zu erhalten gilt, voneinander abgrenzen. Dies ist die Grundlage jeglichen politischen Handelns und die innere Ordnung zu erhalten gegen ein als feindlich empfundenes Außen. So gesehen dient das Politische nicht nur für die Verteidigung der Ordnung und des Staatsfriedens, sondern wird auch für die Eleminierung des Feindes gebraucht. Daher ist jede konkrete politische Ordnung "mit der potentiellen Freund-FeindKonstellation unter Einschluss der möglichen Ausnahmelage gewaltsamer Konflikte konfrontiert" (Vad 1996: 53-54).

Schmitt engt die Rechtfertigung des Krieges auf das Bewahren der eigenen nationalen Souveränität und freiheitlichen Ordnung ein (Vad 1996: 75). Der kriegerische Konflikt als der Ausnahmefall hat im Denken Carl Schmitts eine entscheidende Bedeutung, weil er "die äußerste Konsequenz der politischen Gruppierung von Freund und Feind" darstellt. Daher beinhaltet das Politische, besser gesagt Schmitt's "Freund-Feind-Theorie" auch die Möglichkeit, den Feind praktisch zu zerstören. "Von dieser extremsten Möglichkeit her, gewinnt das Leben der Menschen seine spezifische politische Spannung” (Schmitt 1998: 35). Die permanente Möglichkeit des Konfliktes ist das Fundament seiner politischen Theorie. Nicht der Frieden, sondern seine permanente Gefährdung macht für ihn die elementare Realität der geschichtlichpolitischen Welt aus. Dahinter steht die Erkenntnis von Thomas Hobbes, dass Krieg nicht nur Aktion, sondern eine grundlegende Unsicherheit gegenüber jederzeit möglicher Gewaltanwendung ist (Hobbes 2003: 64; Vad 1996: 83).

Nach diesem Verständnis, das Schmitts Grundgedanken darstellt, braucht die Politik ein "Feindbild", dem er immer widersprechen kann. Dieses Feindbild kann auf religiösen, wirtschaftlichen oder moralischen Ursachen beruhen. Wichtig sind nicht die Gründe, sondern ob sie die Dichte an der politischen Trennung erreichen oder nicht. Aber wann diese Dichte erreicht ist, ist das kein klares Thema (Strauss 1932). Schmitt, als ein Theoretiker des Ausnahmezustandes wurde zum Opfer von dessen Unbeherrschbarkeit (Tucker 1998: 101-102). 
Die ostentative Herausstellung der "auctoritas" dient hier freilich nicht der Beschwörung einer Vergangenheitsidylle, sondem als Spiegel, in dem die an der "veritas" krankende Modeme sich nur mit abgründigem Schaudern wiederfindet (Tucker 1998: 103). Dolf Sternberger fand Schmitt aber "mehr Geschmack an der Ausnahme als an der Norm [...], mehr an der Feindschaft als an der Freundschaft" (Sternberger 1985). In diesem Sinne wurde Schmitt als Nationalist und als Etatist kritisiert. Weil er die Weimarer Republik in Frage gestellt und dem Nationalsozialismus mehr zugeneigt war, wurde er als Nationalist kritisiert (Vad 1996: 11-12). Weil er die Existenz eines mächtigen Staates verteidigte und die Unterscheidung zwischen Feind und Feind ${ }^{10}$ sowohl außen als auch innenpolitisch anwendete, wurde er als Etatist kritisiert (Mehring 2007: 513-518).

Definierte Carl Schmitt den Souveränitätsbegriff und das apologetische Diktum vom Freund-Feind-Theorem als Definitionsmerkmal jeglichen politischen Handelns, so geschah dies in historischer Perspektive vor dem Hintergrund einer staatlichen deutschen Politik in Zeiten der Weimarer Republik: in der staatliches Handelns aufgrund der Divergenz partikularer Parteien und Splitterparteien die Demokratie unterminiert und zur Kulminierung im nationalsozialistischen Umsturz des demokratischen Systems geführt hatte und gifelte, als Machtergreifung deklariert, in der Abschaffung der Demokratie. Die Faschisten hatten hierbei genau diesen Souveränitätsgedanken aufgegriffen, indem sie diesen bedroht sahen, in Anspruch genommen um ihre unrechtliche Praxis politisch zu legitimieren. Die multiple Form demokratischer Willensbildung in Form einer Vielzahl von Parteien war zu einem Gesangsverein degradiert. Zwölf Jahre Nazi-Schreckensherrschaft und einen Weltkrieg später, der Millionen unschuldiger Opfer gefordert hatte, ist der staatliche Souveränitätsgedanke nach wie vor das Hauptcharakteristikum jeglicher politischer Ordnung. Das dialektische Prinzip gilt nicht nur für die Vernunft ( Adorno), es gilt ebenso für die politische Ordnung per se, denn mit diesem Souveränitätsgedanken lassen sich Diktaturen aber auch gerechte demokratische Staaten fundieren. In

\footnotetext{
${ }^{10}$ Nach dem II. Weltkieg definierte Schmitt den "Feind als eigne Frage in Gestalt".
} 
modernen und metamodernen Zeiten wie diesen, in denen sich der Verfasser wiederfindet, ist die politische Praxis nicht mehr wie vor hundert Jahren von vornehmlich miteinander agierenden Nationalstaaten geprägt. Vielmehr bestimmen heute aufgrund des Globalisierungsprozesses wirtschaftliche Interssen politisches Handeln, darauf hatte auch bereits schon Schmitt in seiner vehementen Liberalismuskritik hingewiesen. Dies ist auch heute mehr denn je der Fall.

Nicht nur Globalisierung unterminiert den klassichen Souveränitätsgedanken eines starken Staates, der in seiner Entscheidungsfreiheit nur sich selbst verpflichtet sein soll, um handlungsfähig zu sein. Partikulare Interessen agieren nicht mehr länger nur auf nationaler- parlamentarischer Ebene, wie zu Zeiten der Weimarer Republik Mittlerweile sind paritkulare Interessen als politische Aktuere auf internationler Ebende in Form von NGO's (non governmental organizations), die als gesellschaftliche Interessenverbände konstituiert, über nationalstaatliche Grenzen hinaus, sich international formieren und auf globaler Ebene operieren eine weiterer Faktor, der die Handlungsfähigkeit des souveränden Nationalstaates unterminiere und auf staatliche Entscheidungsprozesse Einfluss nehmen. Staaten im modernen Politikverständnis operieren dabei auch zunehmend auf der Ebene von Staatenbünden (EU), in denen sich der souveräne Einzelstaatswille in einer Mehrzahl von Staaten bündelt und dem Gemeinschaftsprinzip unterordnen muss. Der klassiche Nationalstaat, der sich aus dem Gedanken einer Nation qua gemeinsam geteilter Werte im Sinne von Kultur, Sprache und Religion fundierte, muss sich neu definieren im Angesicht von gesamtgesellschaftlichen Wandlungsprozessen, die durch massive Flüchtlingsströme vor neue Aufgaben gestellt ist (Luhmann 2000: 197-219).

\section{Zusammenfassung}

Thema des vorliegenden Aufsatzes ist die Konzeption des Souveränitätsbegriffs aus diachronischer Sicht beginnend mit der Antike anhand der platonischen und aristotelischen Staatsauffassung über die neuzeitliche Auslegung von Hobbes und Bodin 
bis hin zu dem modernen Staatsrechtler der Weimarer Republik Carl Schmitt. Während Platon und Aristoteles die Aufgabe des Souveräns in der Handlungsweise eines Souveräns im Sinne des gemeinschaftlichen Wohls (to koinon agathon) sahen, geht es bei Bodin und Hobbes in erster Linie darum in Zeiten der Bedrohung der sozialen und politischen Lebensform überhaupt die Bedingungen der Möglichkeit politischer Lebensform zu begründen, um die politische Gemeinschaft $\mathrm{zu}$ konstituieren. Souveränität kann hier nur einer personalisierten Instanz- einem starken Monarchzukommen. Auctoritas und potestas fallen in eins.

Carl Schmitt sieht diese Form der Souveränität in den Zeiten moderner Staatlichkeit als Zeitalter liberaler Entpolitisierung mehr denn je bedroht. Auctoritas und Potestas/Autorität und Macht fallen nicht länger zusammen. Die Souveränität wird als solche negiert. Doch dabei stellt das grundlegende Prinzip der Entscheidung gerade die fundamentale Kategorie staatlicher Souveränität dar. Die Schmittianische FreundFreind Unterscheidung konstituiert eine politische Ordnung im Innern und einem Feind nach Aussen. Auf diese Weise ist der Staat dazu fähig und in der Lage, eine politische Ordnung innerhalb seines definierten Rahmens zu schaffen, in Abgrenzung von einem als feindlich empfundenen Aussen. Dies ist die Grundlage jeglichen politischen Handelns und der inneren Ordnung. Ist der Schmittianische Souveränitätsgedanke vor dem Hintergrund der Weimarer Republik formuliert, historisches Produkt in der Auseinandersetzung mit einer spezifischen geschichtlichen Situation das kennzeichnende Merkmal eines handlungsfähigen Staates, so ist auch die moderne staatliche Handlungspraxis auf dieses Prinzip verwiesen: für eine gerechte und legitime Lebensordnung ihrer Staatsbürger $\mathrm{zu}$ sorgen im Angesicht neuer historischer Herausforderungen. 


\section{LITERATUR} Verlag.

ARISTOTELES (1995). Politik, übst. v. Eugen Rolfes, Hamburg: Felix Meiner

BODIN, Jean (1981). Sechs Bücher über den Staat (1576), Buch I bis III, übersetzt und mit Anmerkungen versehen von Bernd Wimmer, eingeleitet und herausgegeben von Peter Cornelius Mayer-Tasch, München: C. H. Beck Verlag.

GEERLINGS, Wilhelm (2002). Augustinus - Leben und Werk. Eine bibliographische Einführung München: Ferdinand Schöningh Verlag.

HOBBES, Thomas (2003). Der Leviathan. Erster und Zweiter Teil, übrs. v. Jacob Peter Mayer, Stuttgart: Reclam Verlag.

JELLINEK, Georg (1959). Allgemeine Staatslehre (1900), Darmstadt: Wissenschaftliche Buchgesellschaft.

LIETZMANN, Hans (1998). Vater der Verfassungsvater? Carl Schmitt und die Verfassungsgründung in der Bundesrepuhlik, in: Klaus Hansen/Hans Lietzmann (Hrsg.), Carl Schmitt und die Liberalismuskritik (ss. 107-117), Opladen: Leske + Budrich.

LUHMANN, Niklas (2000). Die Politik der Gesellschaft, Frankfurt am Main: Shurkamp Verlag.

MACPHERSON, Crawford Brough (1990). The political Theory of Possessive Individualism. Hobbes to Locke, Oxford: Wynford Books.

MÄDER, Werner (2007). Vom Wesen der Souveränität. Ein deutsches und ein europäisches Problem, Berlin: Duncker \& Humblot Verlag.

MEHRING, Reinhard (2007). Der Begriff des Politischen (1927), in: Manfred von Brocker (Hrsg.), Geschichte des politischen Denkens, (ss. 513-518), Frankfurt am Main: Shurkamp Verlag.

MEHRING, Reinhard (2011). Carl Schmitt zur Einführung, Hamburg: Junius Verlag.

MEIER, Heinrich (1998). Carl Schmitt, Leo Straus und der Begriff des Politischen. Zu einem Dialog unter Abwesenden, Stuttgart: J.B. Metzler Verlag.

PLATON (1998). Der Staat, übst. v. Otto Apelt, Hamburg: Felix Meiner Verlag.

QUARITSCH, Helmut (1986). Souveränität: Entstehung und Entwicklung des Begriffs, Berlin: Duncker \& Humblot Verlag.

QUARITSCH, Helmut (1991). Positionen und Begriffe Carl Schmitts, Berlin: Duncker \& Humblot Verlag.

ROSENBERG, Arthur (1983). Entstehung und Geschichte der Weimarer Republik, Frankfurt am Main: Europäische Verlagsanstalt. 
SCHMITT, Carl (1963). Theorie des Partisanen. Zwischenbemerkung zum Begriff des Politischen, Berlin: Duncker \& Humblot Verlag.

SCHMITT, Carl (1996). Politische Theologie, Vier Kapiteln zur Lehre der Souveränität, 4. Aflg., Berlin: Duncker \& Humblot Verlag.

SCHMITT, Carl (1998). Der Begriff des Politischen, 6. Aflg., Berlin: Duncker \& Humblot Verlag.

SIMON, Rupert (2008). Die Begriffe des Politischen bei Carl Schmitt und Jacques Derrida, Frankfurt am Main: Peter Lang Verlag.

SINGER, Alex (1993). Nationalstaat und Souveränität: Nationalstaat und Souveränität: Zum Wandel des europäischen Staatensystems, Frankfurt am Main: Peter Lang Verlag.

STERNBERGER, Dolf (1985). Irrtümer Carl Schmitts, in: Frankfurter Allgemeine Zeitung, 1. 6. 1985.

STRAUSS, Leo (1932). “Anmerkungen zu Carl Schmitt. Der Begriff des Politischen", Archiv für Sozialwissenschaft und Sozialpolitik, Band 67, Heft 6, pp. 732749.

TUCKER, Bernard (1998). "Der Ausnahmezustand. An den Grenzen von AufkLärung und Liberalismus", in: Klaus Hansen, Hans Lietzmann (Hrsg.), Carl Schmitt und die Liberalismuskritik (ss. 91-105), Opladen: Leske + Budrich.

VAD, Erich (1996). Strategie und Sicherheitspolitik: Perspektiven im Werk von Carl Schmitt, Opladen: Westdeutscher Verlag.

WALDSTEIN, Thor (2008). Der Beutewert des Staates: Carl Schmitt und der Pluralismus, Graz: Ares Verlag.

ZIPPELIUS, Reinhold (2003). Geschichte der Staatsideen, München: C.H.Beck Verlag. 